IDDF2021-ABS-0068 REMOVAL OF HOST DNA FROM TISSUE BIOPSIES IMPROVES DETECTION SENSITIVITY OF GUT MICROBIOTA BY SHOTGUN METAGENOMIC SEQUENCING

Wing-Yin Cheng ${ }^{*}$. The Chinese University of Hong Kong, Hong Kong

10.1136/gutjnl-2021-IDDF.28

Background Shotgun metagenomic sequencing has proved its usefulness in characterizing the microbial species; however, its sensitivity is reduced by the high background of host DNA of tissue biopsies. We aim to examine the effect of host DNA depletion on metagenomic analysis of tissue biopsies.

Methods Human colon biopsies or mouse colon tissues were divided into two groups, with one group undergoing host DNA depletion while the other group serving as the control. Host DNA was removed through differential lysis of mammalian and bacterial cells. The microbial profile of individual samples was determined by metagenomic sequencing and the impacts of host DNA depletion on microbial composition were compared base on phylogenetic diversity analyses and regression analyses.

Results The metagenomic data from human colon samples revealed that the removal of host DNA enhanced bacterial sequencing depth and improved species discovery. Our method increased the number of bacterial reads by $2.46 \pm 0.20$ fold while reducing host reads by $6.80 \pm 1.06 \%$. Moreover, 2998 \pm 401 (3.40 times more) bacterial species were detected after host DNA depletion, whilst only $891 \pm 98$ species were detected in the non-depleted group. This was confirmed in the metagenomic data from mouse colon tissues, showing increased bacterial reads by $5.46 \pm 0.42$ fold while decreasing host reads by $10.2 \pm 0.83 \%$. A total of $2757 \pm 277$ bacterial species were detected after host DNA depletion, whilst only $1154 \pm 53$ species were detected in the non-depleted group. Furthermore, an increased alpha diversity was evident in the host DNA-depleted samples compared to non-depleted controls in human biopsies $(P=0.00021)$ and in mouse colon samples $\left(P=1.2 \times 10^{-7}\right)$.

Conclusions We described an optimized method that removes host DNA from colon tissue and improves the sensitivity of shotgun metagenomic sequencing in bacterial detection in biopsy tissue. This method may yield a more accurate taxonomic profile of the gut microbiota and identify bacteria that are important for disease initiation or progression.

\section{IDDF2021-ABS-0069 ECOLOGY AND NETWORK ANALYSES FOR THE FECAL MICROBIOME IN ULCERATIVE COLITIS (UC)}

Yang Sun*, Yinglei Miao. First Affiliated Hospital of Kunming Medical University, Yunnan Institute of Digestive Diseases, China

\subsection{6/gutjnl-2021-IDDF.29}

Background The incidence of IBD in China (about 3. 44/ 100,000) ranks first among Asian countries. The most difficult thing is that its pathogenesis is still unclear, especially the role of the gut bacteria in host intestinal immune development and defense. This study aimed to analyze the changes in the fecal microbiome associated with UC from the perspectives of medical ecology and complex network analysis.

Methods We analyzed 56 fecal microbiome from 28 Chinese UC patients and their mates, consecutively enrolled at the First
Affiliated Hospital of Kunming Medical University between 2018 to 2019. The effects of UC on the species diversity, species composition and interspecies interaction of fecal microbiome were analyzed and compared at three levels: the community as a whole, the main flora, and the core/peripheral species.

Results After amplicon sequencing and bioinformatics analyzing, in total, 1130 OTUs were identified, in which there were 632 and 666 OTUs in the fecal microbiome of the healthy and UC groups, respectively. In fecal microbiome, the diversity (at diversity order $\mathrm{q}=0$ ) of Bacteroidetes in fecal microbiome was significantly higher in the healthy group than UC group (table 1). And there is a significant difference between their microbial compositions (table 2). For the fecal mucosa, the $\mathrm{P} /$ $\mathrm{N}$ ratio of the healthy group was similar to that of the UC group (figure 1 2). With both $\mathrm{A} 1$ and $\mathrm{A} 2$ algorithms, the observed number of shared core/periphery species was significantly smaller than that expected by chance (table 4).

Conclusions In our study, we innovatively proposed that Hill number for the gut microbe diversity. Then in order to detect whether there was a statistical difference in core (or periphery) species composition between the healthy and UC groups or between the fecal and mucosa microbiome, we adopted a shared core/periphery network (CPN) analysis. Understanding the microbial characteristics and interactions of patients with ulcerative colitis may provide a mechanistic explanation for ulcerative colitis susceptibility and optimize treatment and prevention measures based on individual differences. So this can be a key foundation for precision medicine.

\section{IDDF2021-ABS-0072 P311 PROMOTES M2 MACROPHAGE POLARIZATION AND INFILTRATION IN PANCREATIC DUCTAL ADENOCARCINOMA}

${ }^{1}$ Fangfang Duan*, 'Muzi Ouyang, ${ }^{2}$ Dongwei Jia, ${ }^{1}$ Kongyang Ma, ${ }^{1} \mathrm{ji}$ 'an Pan. ${ }^{1}$ Sun Yat-sen University, China; ${ }^{2}$ Shanghai University of Traditional Chinese Medicine, China

\subsection{6/gutjnl-2021-IDDF.30}

Background Pancreatic ductal adenocarcinoma (PDAC) is one of the most aggressive and lethal malignancies. Treatments such as surgical resection, radiation and chemotherapy have limited efficiency due to the dense stromal and immunosuppressive tumor microenvironment (TME). The interaction between the microenvironment and cancer cells remains to be further elucidated. In this study, we characterized the prognostic value of P311 in PDAC and aimed to examine the interaction between tumor-derived P311 and M2 macrophage and investigate the underlying mechanisms.

Methods Expression datasets of PDAC patients were downloaded from The Cancer Genome Atlas (TCGA) and Gene Expression Omnibus (GEO) databases. The immune score was calculated by applying the ESTIMATE algorithm to the matrix data. The deconvolution algorithm (CIBERSORT) was used to assess 22 tumor-infiltrating immune cells (TIICs) for their amounts in the PDAC microenvironment. Stable PANC-1 cells expressing P311 were generated from lentiviral particles. RNA sequencing (RNA-Seq) was conducted to investigate geneexpression profiling from P311 overexpressed PANC-1 cells. The association between P311 expression and M2 macrophage polarization was determined by flow cytometry.

Results P311 was overexpressed in tissues of PDAC patients compared with the adjacent normal tissues and associated with poor overall survival (OS). Based on ESTIMATE algorithm, P311 positively correlated with ESTIMATE score and stromal 